\title{
The gravitino problem in extended gravity cosmologies
}

\author{
Salvatore Capozziello $^{1,2,3,4, \mathrm{a}}{ }_{\mathbb{D}}$, Gaetano Lambiase $\mathbf{L}^{5,6, \mathrm{~b}}$ \\ ${ }^{1}$ Dipartimento di Fisica "E. Pancini”, Università di Napoli "Federico II", Via Cinthia, 80126 Napoli, Italy \\ 2 Istituto Nazionale di Fisica Nucleare (INFN), Sez. di Napoli, Via Cinthia, 80126 Napoli, Italy \\ ${ }^{3}$ Scuola Superiore Meridionale, Largo San Marcellino 10, 80138 Napoli, Italy \\ ${ }^{4}$ Laboratory for Theoretical Cosmology, Tomsk State University of Control Systems and Radioelectronics \\ (TUSUR), Tomsk, Russia 634050 \\ 5 Dipartimento di Fisica E.R. Cainaiello, University of Salerno, Via Giovanni Paolo II, 84084 Fisciano, SA, \\ Italy \\ ${ }^{6}$ INFN, Gruppo Collegato di Salerno, Sezione di Napoli, Via Giovanni Paolo II, 84084 Fisciano, SA, Italy
}

Received: 24 December 2020 / Accepted: 11 January 2021

(C) The Author(s) 2021

\begin{abstract}
The gravitino problem is investigated in the framework of extended gravity cosmologies. In particular, we consider $f(R)$ gravity, the most natural extension of the HilbertEinstein action, and $f(\mathcal{T})$ gravity, the extension of teleparallel equivalent gravity. Since in these theories, the expansion laws of the Universe are modified, as compared to the standard $\Lambda \mathrm{CDM}$ cosmology, it follows that also the thermal history of particles gets modified. We show that $f(R)$ models allow to avoid the late abundance of gravitinos. In particular, we found that for an appropriate choice of the parameters characterizing the $f(R)$ model, the gravitino abundance turns out to be independent of the reheating temperature. A similar behavior is achieved also in the context of $f(\mathcal{T})$ gravity. In this perspective, we can conclude that geometric corrections to standard General Relativity (and to Teleparallel Equivalent of General Relativity) can improve shortcomings both in cosmology and in unified theories beyond the standard model of particles.
\end{abstract}

\section{Introduction}

One of the most attractive extensions of the standard model of particles (SM) is represented by supersymmetry (SUSY) [1] since it provides the stability of electroweak scale against radiative corrections and since the three gauge couplings of the SM meet at GUT scales $10^{16} \mathrm{GeV}$. Apart these motivations, there is, up to now, no direct evidence of the existence of superpartner particles predicted by the theory. A natural extension of SUSY is the supergravity (SUGRA), which is essentially the local counterpart of SUSY [2]. The SUGRA models predict the existence of the gravitino (the superpartner of the graviton). The latter, in some SUSY models $[3,4]$, is characterized by the fact that its mass is not directly related to the SUSY breaking scales of SM ordinary particles and their superpartners. It is worth noticing that its interaction is weak, so that the chance to find it in collider experiments is extremely

Focus Point on Modified Gravity Theories and Cosmology Guest editors: S. Capozziello, V. Gurzadyan.

\footnotetext{
a e-mail: capozziello@na.infn.it (corresponding author)

b e-mail: lambiase@sa.infn.it
}

Published online: 29 January 2021 
unlikely. On the other hand, the gravitino can have interesting consequences at cosmological scales, in particular during the early phases of the Universe evolution.

An open issue in cosmology is the so-called gravitino problem [5-8]. Since SUSY particles couple to (ordinary) matter only through the gravitational interaction, it turns out that their couplings are Planck suppressed, implying a quite long lifetime

$$
\tau \sim \frac{M_{P l}^{2}}{m_{3 / 2}} \simeq 10^{5}\left(\frac{1 \mathrm{TeV}}{m_{3 / 2}}\right)^{3} \mathrm{~s},
$$

where $m_{3 / 2}$ is the gravitino mass. The scale characterizing $m_{3 / 2}$ is of the order of electroweak energies $\mathcal{O} 10^{2} \mathrm{GeV}$. One of the consequences of particles with so long lifetime is that if they decay after the Big Bang nucleosynthesis (BBN), then their decaying products would destroy light elements, and hence in contradiction with the successful predictions of BBN. This problem is avoided by setting an upper bound on the reheating temperature. In the framework of General Relativity (GR), the gravitino abundance $Y_{3 / 2}$ is proportional to the reheating temperature $T_{R}[9]$

$$
Y_{3 / 2} \simeq 10^{-11} \frac{T_{R}}{10^{10} \mathrm{GeV}}
$$

so that requiring that it remains small $Y_{3 / 2} \lesssim 10^{-14}$ for a successful prediction of BBN, one gets the constraint $[10,11]$

$$
T_{R} \lesssim\left(10^{6}-10^{7}\right) \mathrm{GeV} \text { for } m_{3 / 2} \sim \mathcal{O}\left(10^{2} \mathrm{GeV}\right) .
$$

The bound (3) opens some issues related to the inflationary scenarios since the latter provides a reheating temperature larger than the upper bound (3) (see [12-18]).

In this paper, we consider the gravitino problem in the framework extended gravity cosmologies. As possible models of extended gravity, we shall consider $f(R)$ theory, which represents the simplest extension of theories involving the Ricci curvature scalar $R$, and the $f(\mathcal{T})$ theory, where $\mathcal{T}$ is the torsion field adopted in the Teleparallel Equivalent of General Relativity (TEGR). These theories have been recently invoked in order to explain the discovery of accelerated expansion of the Universe [19,20] without introducing unknown forms of dark energy, and, in the astrophysical contexts, to fix the dark matter problem. The appearance of these dark components in the Universe dynamics is a clear signal of the breakdown of GR at infrared scales.

The aim of this paper is to show that considering cosmological models related to these theories can contribute to alleviate the above gravitino problem.

The paper is organized as follows. In Sect. 2, we discuss the gravitino problem in the framework of $f(R)$ cosmology. The $f(\mathcal{T})$ cosmology and the gravitino problem are considered in Sect. 3. Discussion and conclusions are reported in Sect. 4.

\section{The gravitino problem in $f(R)$ cosmology}

The gravitational action for $f(R)$ gravity is [21-44]

$$
S=\frac{1}{2 \kappa^{2}} \int d^{4} x \sqrt{-g} f(R)+S_{m}\left[g_{\mu \nu}, \psi_{m}\right]
$$

where $S_{m}$ is the matter action and $\kappa^{2}=8 \pi G=8 \pi / M_{P l}^{2}$ is the gravitational coupling. ( $M_{P l} \simeq 10^{19} \mathrm{GeV}$ is the Planck mass.) The action (4) must be considered as an effective 
theory that allows to describe, at a phenomenological level, the gravitational interactions. The field equations for $f(R)$ gravity are

$$
f^{\prime} R_{\mu \nu}-\frac{f}{2} g_{\mu \nu}-\nabla_{\mu} \nabla_{\nu} f^{\prime}+g_{\mu \nu} \square f^{\prime}=\kappa^{2} T_{\mu \nu}^{m},
$$

while the trace equation is given by

$$
3 \square f^{\prime}+f^{\prime} R-2 f=\kappa^{2} T^{m}, \quad T^{m}=\rho-3 p .
$$

Here, $f^{\prime} \equiv \frac{\partial f}{\partial R}$. In cosmology, it is $\square f^{\prime}=\ddot{f}^{\prime}+3 H \dot{f}^{\prime}, H=\dot{a} / a$, and the dot stands for $d / d t$. $T_{\mu \nu}^{m}$ is the matter energy-momentum tensor and $T^{m}$ its trace. The tensor $T_{\mu \nu}^{m}$ is divergenceless, i.e., $\nabla^{\mu} T_{\mu \nu}^{m}=0$, and this property holds also for the 1.h.s. of (5). There are several hypotheses on the explicit form of function $f(R)$; however, at the moment, there is no final statement on a comprehensive form working at any scale. For the sake of simplicity, we adopt a power law model (for other models and applications, see [21-49])

$$
f(R)=\alpha R^{n},
$$

where $n$ is a real number. Clearly, standard GR is recovered for $n=1$.

In a (spatially flat) Friedman-Lemaitre-Robertson-Walker (FLRW) metric $d s^{2}=d t^{2}-$ $a^{2}(t)\left[d x^{2}+d y^{2}+d z^{2}\right]$, with the ansatz $a(t)=a_{0} t^{\beta}$, the $0-0$ field equation and the trace equation read

$$
\begin{aligned}
\frac{\alpha}{2}\left[\frac{n(\beta+2 n-3)}{2 \beta-1}-1\right] R^{n} & =\kappa^{2} \rho, \\
\alpha\left[n-2-\frac{n(n-1)(2 n-1)}{\beta(1-2 \beta)}+\frac{3 n(n-1)}{1-2 \beta}\right] R^{n} & =\kappa^{2}(1-3 w) \rho,
\end{aligned}
$$

where $\rho$ is the energy density which, in the radiation dominated era, reads

$$
\rho^{m}=\frac{\pi^{2} g_{*}}{30} T^{4},
$$

where $g_{*} \simeq 10^{2}$ counts the number of relativistic degrees of freedom.

In a radiation dominated Universe, where the trace $T^{m}=0$, Eqs. (8) and (9) yield

$$
\alpha \Omega_{n} R^{n}=\kappa^{2} \rho,
$$

where we have defined the quantities

$$
\Omega_{n} \equiv \frac{5 n^{2}-8 n+2}{4(n-1)} \quad \beta=\frac{n}{2} .
$$

From Eq. (11), one obtains the relation between the cosmic time $t$ and the temperature $T$

$$
t=\Pi_{n}\left(\frac{T}{M_{P l}}\right)^{-\frac{2}{n}} M_{P l}^{-1}
$$

here, it is

$$
\Pi_{n} \equiv[3 n|n-1|]^{1 / 2}\left(\frac{15 \tilde{\alpha} \Omega_{n}}{4 \pi^{3} g_{*}}\right)^{\frac{1}{2 n}}, \quad \tilde{\alpha}=\frac{\alpha}{M_{P l}^{2(1-n)}}
$$


Let us introduce the transition time (temperature) $t_{*}\left(T_{*}\right)$ which characterizes the transition from the $f(R)$ cosmology to the standard cosmology, described by GR. This means to equate the equation of the evolution at the instant $t=t_{*}$, i.e., $\alpha \Omega_{\beta, n} R^{n}\left(t_{*}\right)=H_{G R}^{2}\left(t_{*}\right)$. One gets

$$
t_{*}=\left[4 \tilde{\alpha} \Omega_{n}[3 n|n-1|]^{n}\right]^{\frac{1}{2(n-1)}} M_{P l}^{-1},
$$

The expression of the transition temperature $T_{*}$ is given by

$$
T_{*} \equiv M_{P l}[3 n|n-1|]^{-\frac{n}{4(n-1)}}\left[\frac{15}{16 \pi^{3} g_{*}}\right]^{\frac{1}{4}}\left[4 \tilde{\alpha} \Omega_{n}\right]^{-\frac{1}{4(n-1)}},
$$

so that relation (15) can be cast in the form

$$
t=t_{*}\left(\frac{T}{T_{*}}\right)^{-\frac{2}{n}} .
$$

Moreover, notice that

$$
\frac{t_{*} T_{*}^{2}}{M_{P l}}=\sqrt{\frac{15}{16 \pi^{3} g_{*}}} .
$$

In what follows, we shall assume that, after inflation, from GUT scales to reheating scales, the Universe evolves according to (7), and then, its evolution enters in the regime governed by the cosmological standard model. Hence, the $f(R)$ cosmology provides a scenario in which the thermal history of particles gets modified, and as a consequence, the Boltzmann equation too (see below). This reflects on the gravitino abundance, which turns out to depend on parameters characterizing the $f(R)$ model and the transition temperature, i.e., the temperature at which the Universe passes from the $f(R)$ evolution to the standard GR one. In this phase, the parameters $\{\alpha, n\}$ can play an important role.

Let us notice that the expansion rate of the Universe in $f(R)$ cosmology can be written as

$$
\begin{aligned}
H(T) & =A(T) H^{(G R)}(T), \\
A(T) & \equiv \eta\left(\frac{T}{T_{*}}\right)^{p} \\
\text { with } \quad \eta & \equiv 2 \sqrt{3} \beta, \\
p & \equiv \frac{2}{n}-2
\end{aligned}
$$

where $A(T)$ is an enhancement factor. Expressions similar to (19) are obtained in different frameworks: $p=2$ in Randall-Sundrum type II brane cosmology [50], $p=1$ in kination models [51-53], $p=-1$ in scalar-tensor cosmology [54], $-1 \lesssim p \lesssim 0$ in various $f(R)$ cosmologies [55].

As pointed out in Introduction, gravitino is generated by means of thermal scattering in the primordial plasma. This occurs during the reheating era after inflation. To describe the gravitino production, one makes use of the Boltzmann equation for the number density of species in thermal bath. Following Ref. [5], the relevant equation for gravitino production is

$$
\frac{\mathrm{d} n_{3 / 2}}{\mathrm{~d} t}+3 H n_{3 / 2}=\langle\sigma v\rangle n_{\mathrm{rad}}^{2} .
$$

Here, $n_{3 / 2 \text {,rad }}$ refers to gravitino and relativistic species, while $\langle\ldots\rangle$ stands for the thermal average of the gravitino cross section $\sigma v$ times the relative velocity of scattering radiation 
$(v \sim 1)$. In (21), we have neglected the term $\frac{m_{3 / 2}}{\left\langle E_{3 / 2}\right\rangle} \frac{n_{3 / 2}}{\tau_{3 / 2}}$, where $\frac{m_{3 / 2}}{\left\langle E_{3 / 2}\right\rangle}$ is the average Lorentz factor. Introducing the gravitino and relativistic particles abundances $Y_{3 / 2}=n_{3 / 2} / \mathrm{s}$ and $Y_{\mathrm{rad}}=n_{\mathrm{rad}} / s$, respectively, where $s=\frac{2 \pi^{2}}{45} g_{*} T^{3}$ and $g_{*} \sim 300$, the Boltzmann Eq. (21) assumes the form

$$
\frac{\mathrm{d} Y_{3 / 2}}{\mathrm{~d} T}=\frac{s\langle\sigma v\rangle}{\dot{T}} Y_{\mathrm{rad}}^{2}
$$

Here, we used

$$
\frac{\dot{T}}{T}=-\frac{n}{2 t}=-\frac{n}{2 t_{*}}\left(\frac{T}{T_{*}}\right)^{\frac{2}{n}}
$$

Integrating from $T_{R}\left(\gg T_{*}\right.$, see below) up to a low temperature $T_{l}\left(\lesssim T_{*}\right)$ in the era described by GR, the solution to (22) is

$$
Y_{3 / 2} \simeq-\mathcal{B} \frac{M_{P l}}{T_{*}}\left[\left(\frac{T_{R}}{T_{*}}\right)^{\Delta}-\left(\frac{T_{l}}{T_{*}}\right)^{\Delta}\right],
$$

where

$$
\begin{aligned}
\Delta & \equiv 3-\frac{2}{n} \\
\mathcal{B} & \equiv\left[M_{P l} \frac{\langle\sigma v\rangle s}{H_{G R} T} Y_{\text {rad }}^{2}\right]_{R} \frac{1}{\sqrt{3}(3 n-2)} .
\end{aligned}
$$

Notice that (24) is independent of the parameter $\alpha$.

The gravitino abundance derived in Eq. (24) allows to alleviate the gravitino problem. In fact, for $\Delta \approx 0$, i.e., $n \approx 2 / 3$, it follows that the gravitino abundance turns out to be $Y_{3 / 2}<<1$. The most stringent constrain on unstable massive relic particles with lifetime $\gtrsim 10^{2} \mathrm{sec}$, obtained from ${ }^{6} \mathrm{Li}$ abundance, is $Y_{3 / 2} \lesssim 10^{-14} \frac{10^{2} \mathrm{GeV}}{m_{3 / 2}}[10,11]$. Using this value, it follows that the overproduction of gravitino is avoided if the transition temperature is $T_{*} \lesssim\left(10^{6}-10^{7}\right) \mathrm{GeV}$, while the reheating temperature can be larger in order to be compatible with inflationary prediction values.

\section{The gravitino problem in $f(\mathcal{T})$ cosmology}

An interesting approach to gravity is the TEGR, firstly proposed by Einstein himself [57, 58]. This theory of gravity is based on the Weitzenböck connection (instead of the LeviCivita connection), and the gravitational field is described by the vierbein fields $e_{\mu}^{i}(x)$ whose dynamics is governed by the torsion tensor (instead of the curvature tensor), that is

$$
\mathcal{T}_{\mu \nu}^{\lambda}=\hat{\Gamma}_{\nu \mu}^{\lambda}-\hat{\Gamma}_{\mu \nu}^{\lambda}=e_{i}^{\lambda}\left(\partial_{\mu} e_{\nu}^{i}-\partial_{\nu} e_{\mu}^{i}\right)
$$

Here, $e_{\mu}^{i}(x)$ are defined as $g_{\mu \nu}(x)=\eta_{i j} e_{\mu}^{i}(x) e_{\nu}^{j}(x)$. The possible action is given by

$$
S_{I}=\frac{1}{16 \pi G} \int d^{4} x e[\mathcal{T}+f(\mathcal{T})]
$$


where $\mathcal{T}=S_{\rho}{ }^{\mu \nu} \mathcal{T}_{\mu \nu}^{\rho}$ is the torsion scalar, $e=\operatorname{det}\left(e_{\mu}^{i}\right)=\sqrt{-g}, f(\mathcal{T})$ is a generic function of the torsion, and

$$
S_{\rho}^{\mu \nu}=\frac{1}{2}\left[\frac{1}{4}\left(\mathcal{T}_{\rho}^{\mu v}-\mathcal{T}^{v \mu}{ }_{\rho}-\mathcal{T}_{\rho}^{\mu \nu}\right)+\delta_{\rho}^{\mu} \mathcal{T}^{\theta v}{ }_{\theta}-\delta_{\rho}^{\nu} \mathcal{T}^{\theta \mu}{ }_{\theta}\right] .
$$

TEGR represents an alternative to GR where the coincidence between geodesic structure and causal structure is not required. This means that equivalence principle is not at the foundation of the theory, but affine structure is more relevant. In cosmology, the approach revealed extremely useful to address inflation as well as the late accelerated expansion. In this perspective, the dynamical key role is played by torsion instead of curvature [57-60]).

For the above homogeneous, isotropic and spatially flat FLRW Universe, one finds that $e_{\mu}^{A}=\operatorname{diag}(1, a, a, a)$ and $\mathcal{T}=-6 H^{2}$. The cosmological field equations are [60]

$$
12 H^{2}\left[1+f_{\mathcal{T}}\right]+[\mathcal{T}+f]=16 \pi G \rho,
$$

$$
48 H^{2} f_{\mathcal{T} \mathcal{T}} \dot{H}-\left(1+f_{\mathcal{T}}\right)\left[12 H^{2}+4 \dot{H}\right]-(\mathcal{T}-f)=16 \pi G p,
$$

where $f_{\mathcal{T}}=\mathrm{d} f / \mathrm{d} \mathcal{T}$. As an explicit example, we consider the power law $f(T)$ model [61-64]

$$
f(\mathcal{T})=\beta_{\mathcal{T}}|\mathcal{T}|^{n_{\mathcal{T}}},
$$

where $n_{\mathcal{T}}$ has, in principle, another meaning with respect to the above $n$ used in $f(R)$ gravity. By rewriting (30) in the form

$$
H_{T E G R}^{2}+H_{\mathcal{T}}^{2}=\frac{8 \pi}{3 M_{P l}^{2}} \rho
$$

where

$$
H_{\mathcal{T}}^{2} \equiv \frac{f}{6}-\frac{T f_{\mathcal{T}}}{3}=6^{\left(n_{\mathcal{T}}-1\right)} \beta_{\mathcal{T}}\left(2 n_{\mathcal{T}}+1\right) H^{2 n_{\mathcal{T}}},
$$

and assuming $H_{\mathcal{T}} \gg H_{T E G R}$, one gets the expressions as in (19) and (20), with

$$
\begin{aligned}
\eta & =1, \quad v=\frac{2}{n_{\mathcal{T}}}-2 \\
T_{*} & \equiv\left(\frac{24 \pi^{3} g_{*}}{45}\right)^{\frac{1}{4}}\left(2 n_{\mathcal{T}}+1\right)^{\frac{1}{4\left(1-n_{\mathcal{T}}\right)}}\left(\frac{\beta_{\mathcal{T}}}{\mathrm{GeV}^{2\left(1-n_{\mathcal{T}}\right)}}\right)^{\frac{1}{4\left(1-n_{\mathcal{T}}\right)}}\left(\frac{M_{P l}}{\mathrm{GeV}}\right)^{\frac{1}{2}} \mathrm{GeV}
\end{aligned}
$$

or explicitly (see (31))

$$
A(T)=\left(\frac{1}{\left(2 n_{\mathcal{T}}+1\right)} \frac{\mathrm{GeV}^{2\left(1-n_{\mathcal{T}}\right)}}{\beta_{\mathcal{T}}}\right)^{\frac{1}{2 n_{\mathcal{T}}}}\left[\left(\frac{45}{24 \pi^{3} g_{*}}\right)^{\frac{1}{2}}\left(\frac{T}{\mathrm{GeV}}\right)^{2} \frac{\mathrm{GeV}}{M_{P l}}\right]^{\frac{1}{n_{\mathcal{T}}}-1} .
$$

In order to solve the gravitino problem, we have to consider the bound $n_{\mathcal{T}} \leqslant 2 / 3[62,63]$, i.e., $v \geqslant 1$, as imposed by BBN constraints. We shall discuss the case $n_{\mathcal{T}}=2 / 3$, i.e., $v=1$, and $n_{\mathcal{T}}<2 / 3$, i.e., $v>1$.

The gravitino problem is studied by means of the Boltzmann (22). For $v=1$ and $v>1$, the integration of (22) from $T_{*}$ to $T_{R}$ (assuming $T_{R} \gg T_{*}$ and using (31)) gives

$$
Y_{3 / 2} \sim \begin{cases}\frac{T_{*}}{\eta} \log \frac{T_{R}}{T_{*}} & v=1 \\ \frac{T_{*}^{2 v-1}}{\eta(v-1)} & v>1\end{cases}
$$


which is independent of the reheating temperature. Therefore, if the transition temperature is low enough, i.e., $T_{*}<\left(10^{6} \div 10^{7}\right) \mathrm{GeV}$, the gravitino problem is avoided even if the reheating temperature is much higher.

\section{Discussion and conclusions}

Before drawing our conclusions, let us check that the condition $T_{R} \gg T_{*}$ occurs also in non-standard cosmology. This result applies to the above models of modified cosmologies. By comparing the expansion rate of the Universe (19) with the decay rate of the inflaton $\Gamma_{I}$, one obtains the reheating temperature

$$
T_{R}=T_{*}\left(\frac{M_{P l} \Gamma_{I}}{T_{*}^{2}}\right)^{\frac{1}{v+2}} .
$$

In the standard cosmology, the relation $H_{G R}\left(T_{R}^{G R}\right)=\Gamma_{I}$ gives $T_{R}^{G R} \simeq\left(M_{P l} \Gamma_{I}\right)^{1 / 2}$. Combining this relation with the reheating temperature (33), one infers

$$
T_{R}=T_{*}\left(\frac{T_{R}^{G R}}{T_{*}}\right)^{\frac{2}{v+2}} .
$$

Thus, $T_{R} \gg T_{*}$ provided $T_{R}^{G R} \gg T_{*}$. This relation, however, is such that the values $v \gg 1$ are excluded.

To conclude, we have presented some preliminary results related to the gravitino problem assuming that the background is described by modified cosmological models coming from extended gravity. Using the fact that the expansion rate of the Universe gets modified as $H=$ $A(T) H_{G R}$, where the factor $A(T)$ (given in Eq. (19)) accounts for the non-standard evolution of the cosmic background, we solved the Boltzmann equation describing the time evolution of the gravitino abundance. It is possible to show that, under specific condition, modified cosmology can provide natural cosmological scenarios able to avoid the late overproduction of gravitinos. The analysis carried out in this paper relies on models derived from $f(R)$ and $f(\mathcal{T})$ gravities. However, besides the possibility to find more general solutions, other curvature and torsion invariants might play a relevant role for the gravitino problem here studied. In particular, cosmological models of the form $f\left(R, \square R, \square^{l} R, \ldots\right)$ or $f\left(\mathcal{T}, \square \mathcal{T}, \square^{l} \mathcal{T}, \ldots\right)$ deserve to be taken into account $[65,66]$. Such studies will be considered in forthcoming papers.

Acknowledgements The authors are supported by the INFN sezione di Napoli and gruppo collegato di Salerno, iniziative specifiche MOONLIGHT2 and QGSKY.

Funding Open Access funding provided by Universitá degli Studi di Napoli Federico II

Open Access This article is licensed under a Creative Commons Attribution 4.0 International License, which permits use, sharing, adaptation, distribution and reproduction in any medium or format, as long as you give appropriate credit to the original author(s) and the source, provide a link to the Creative Commons licence, and indicate if changes were made. The images or other third party material in this article are included in the article's Creative Commons licence, unless indicated otherwise in a credit line to the material. If material is not included in the article's Creative Commons licence and your intended use is not permitted by statutory regulation or exceeds the permitted use, you will need to obtain permission directly from the copyright holder. To view a copy of this licence, visit http://creativecommons.org/licenses/by/4.0/. 


\section{References}

1. J. Wess, B. Zumino, Nucl. Phys. B 70, 39 (1974)

2. E. Cremmer, S. Ferrara, L. Girardello, A. van Proyen, Nucl. Phys. B 212, 413 (1983)

3. J. Ellis, C. Kounnas, D.V. Nanopoulos, Phys. Lett. B 143, 410 (1984)

4. J. Ellis, K. Enqvist, D.V. Nanopoulos, Phys. Lett. B 147, 99 (1984)

5. N. Okada, O. Seto, Phys. Rev. D 73, 063505 (2006)

6. M.Y. Khlopov, A.D. Linde, Phys. Lett. B 138, 265 (1984)

7. J.R. Ellis, J.E. Kim, D.V. Nanopoulos, Phys. Lett. B 145, 181 (1984)

8. M. Kawasaki, K. Kohri, T. Moroi, Phys. Lett. B 625, 7 (2005)

9. M. Kawasaki, T. Moroi, Prog. Theor. Phys. 93, 879 (1995)

10. R.H. Cyburt, J.R. Ellis, B.D. Fields, K.A. Olive, Phys. Rev. D 67, 103521 (2003)

11. M. Kawasaki, K. Khori, T. Moroi, Phys. Lett. B 625, 7 (2005)

12. K.A. Olive, Phys. Rep. 190, 307 (1990)

13. D.H. Lyth, A. Riotto, Phys. Rep. 314, 1 (1999)

14. A. Mazumdar, Phys. Rev. D 64, 027304 (2001)

15. A. Mazumdar, Nucl. Phys. b 597, 561 (2001)

16. M.C. Bento, N.C. Santos, R.G. Felipe, Phys. Rev. D 69, 123513 (2004)

17. N. Okada, O. Seto, Phys. Rev. D 71, 023517 (2005)

18. N. Okada, O. Seto, Phys. Rev. D 73, 063505 (2006)

19. A.G. Reiss et al., Astron. J. 116, 1009 (1998)

20. S. Perlmutter et al., Nature (London) 391, 51 (1998)

21. L. Amendola, S. Tsujikawa, Dark Energy: Theory and Observations (Cambridge University Press, Cambridge, 2010)

22. S. Capozziello, Int. J. Mod. Phys. D 11, 483 (2002)

23. S. Capozziello, M. De Laurentis, Phys. Rep. 509, 167 (2011)

24. S. Nojiri, S.D. Odintsov, Phys. Rep. 505, 59 (2011)

25. A. Silvestri, M. Trodden, Rep. Prog. Phys. 72, 096901 (2009)

26. J.A. Frieman, M.S. Turner, D. Huterer, Annu. Rev. Astron. Astrophys. 46, 385 (2008)

27. R. Durrer, R. Maartens, Gen. Relat. Grav. 40, 301 (2008)

28. S. Capozziello, G. Lambiase, Frascati Phys. Ser. 58, 17 (2014)

29. M. Sami, Lect. Notes Phys. 720, 219 (2007)

30. E.J. Copeland, M. Sami, Sh. Tsujikawa, Int. J. Mod. Phys. D 15, 1753 (2006)

31. T. Clifton, P.G. Ferreira, A. Padilla, C. Skordis, Phys. Rep. 513, 1 (2012)

32. T.P. Sotiriou, V. Faraoni, Rev. Mod. Phys. 82, 451 (2010)

33. A. De Felice, S. Tsujikawa, Living Rev. Rel. 13, 3 (2010)

34. S. Nojiri, S.D. Odintsov, Phys. Rev. D 77, 026007 (2008)

35. H. Oyaizu, M. Lima, W. Hu, Phys. Rev. D 78, 123524 (2008)

36. L. Pogosian, A. Silvestri, Phys. Rev. D 77, 023503 (2008)

37. I. Sawicki, W. Hu, Phys. Rev. D 75, 127502 (2007)

38. B. Li, J.D. Barrow, Phys. Rev. D 75, 084010 (2007)

39. T. Clifton, Phys. Rev. D 78, 083501 (2008)

40. S. Capozziello, G. Lambiase, Gen. Relat. Gravit. 32, 295 (2000)

41. S. Capozziello, G. Lambiase, Gen. Relat. Gravit. 31, 1005 (1999)

42. T. Clifton, J.D. Barrow, Phys. Rev. D 72, 103005 (2005)

43. W. Hu, I. Sawicki, Phys. Rev. D 76, 064004 (2007)

44. A.A. Starobinsky, JETP Lett. 86, 157 (2007)

45. S. Capozziello, M. de Laurentis, G. Lambiase, Phys. Lett. B 715, 1 (2012)

46. G. Lambiase, Phys. Rev. D 90, 064050 (2014)

47. S. Capozziello, G. Lambiase, M. Sakellariadou, A. Stabile, A. Stabile, Phys. Rev. D 91, 044012 (2015)

48. G. Lambiase, S. Mohanty, A.R. Prasanna, Int. J. Mod. Phys. D 22, 1330030 (2013)

49. G. Lambiase, L. Mastrototaro, Astrophys. J. 904:19, 1 (2020)

50. L. Randal, R. Sundrum, Phys. Rev. Lett. 83, 4690 (1991)

51. F. Profumo, P. Ullio, JCAP 0311, 006 (2003)

52. F. Rosati, Phys. Lett. B 570, 5 (2003)

53. C. Pallis, JCAP 0510, 015 (2005)

54. R. Catena, N. Fornengo, A. Masiero, M. Pietroni, F. Rosati, Phys. Rev. D 70, 063519 (2004)

55. S. Capozziello, V. Galluzzi, G. Lambiase, L. Pizza, (n.d.) arXiv:1507.06835 [astro-ph.CO]

56. A. Conroy, A. Mazumdar, A. Teimouri, Phys. Rev. Lett. 114, 201101 (2015)

57. R. Ferraro, F. Fiorini, Phys. Rev. D. 75, 084031 (2007) 
58. R. Aldrovandi, J.G. Pereira, Teleparallel Gravity: An Introduction (Springer, Dordrecht, 2013)

59. E.V. Linder, Phys. Rev. D 81, 127301 (2010)

60. Y.F. Cai, S. Capozziello, M. De Laurentis, E.N. Saridakis, Rep. Prog. Phys. 79(10), 106901 (2016)

61. S. Nesseris, S. Basilakos, E.N. Saridakis, L. Perivolaropoulos, Phys. Rev. D 88, 103010 (2013)

62. S. Capozziello, G. Lambiase, E.N. Saridakis, Eur. Phys. J. C 77, 576 (2017)

63. M. Benetti, S. Capozziello, G. Lambiase, Mon. Not. R. Astron. Soc. 500, 1795 (2021)

64. R. Ferraro, F. Fiorini, Phys. Rev. D 78, 124019 (2008)

65. S. Capozziello, M. Capriolo, L. Caso, Class. Quantum Gravity 37, 235013 (2020)

66. S. Capozziello, M. Capriolo, S. Nojiri, Phys. Lett. B 810, 135821 (2020) 